\title{
La discapacidad, las barreras físicas y el ejercicio ciudadano en la lucha por el derecho a la ciudad en Medellín, Colombia (2007- 2015)*
}

\author{
Disability, Physical barriers and the citizenship exercise in the struggle for the \\ right to the city in Medellín, Colombia (2007-2015)
}

James Larry Vinasco Hernández ${ }^{* *}$

\section{Resumen}

El artículo analiza los hallazgos más importantes en la identificación de las tensiones y acuerdos entre las organizaciones de personas con discapacidad y la administración municipal de Medellín en la planeación de ciudad, con el fin de comprender las formas de incidencia de estas organizaciones frente al derecho a la ciudad. En este contexto se generan distintas estrategias ciudadanas frente a la planeación y el diseño de espacios físicos accesibles derivadas de su práctica espacial y en la construcción social de la discapacidad. Desde este conjunto de hechos, se formulan los elementos fundamentales para entender la importancia de la discapacidad como forma particular de construir ciudad por medio del ejercicio ciudadano en búsqueda de la inclusión social y la garantía de derechos de esta población.

\footnotetext{
* Artículo recibido 26 de septiembre de 2016. Aceptado 1 de febrero de 2017.

** Sociólogo, maestrante en Sociología de la Universidad de Antioquia, Colombia. Correo electrónico: james.vinasco@udea.edu.co, jlarry2@gmail.com
} 


\section{Abstract}

The article analyses the most important findings in the identification of tensions and agreements between organizations of persons with disabilities and the municipal administration of Medellin in city planning, in order to understand the ways of incidence of these organizations for the right to the city. In this context, different citizen strategies is generated in relation to the planning and design of accessible physical spaces derived from their spatial practice and the social construction of disability. From this set of facts, the fundamental elements are formulated to understand the importance of disability as a particular way of building a city through the exercise of citizenship in search of social inclusion and the guarantee of rights.

\section{Palabras clave}

Discapacidad-Derecho a la ciudad-Medellín (Colombia).

\section{Keywords}

Disability-Right to the city-Medellín (Colombia).

\section{Elementos introductorios y de contexto}

Este artículo aborda como fundamento los elementos compositivos de la discapacidad como hecho social a partir de las perspectivas de las personas involucradas en el mismo en la relación planeación de ciudad y discapacidad. En un primer apartado se desarrolla la introducción al tema específico, señalando las discusiones principales incluidas en el artículo; seguido, se hará una contextualización de la temática en cuanto a los hallazgos principales en el acercamiento al fenómeno a través de diálogos y observaciones en campo. Posteriormente, se mirarán los resultados principales analizados y relacionados a través de algunas categorías orientadoras $y$, en último lugar, están las conclusiones generales acerca de la fuerza que ha tomado en la ciudad la discapacidad como forma de entender la participación ciudadana en la planeación participativa e incluyente de la misma.

Por ello, privilegiar las acciones de las personas con discapacidad es una forma de encontrar varios elementos nuevos o poco abordados para el contexto de la ciudad de Medellín ${ }^{1}$. Por un lado, nos permite entender la relación entre distintos actores que

\footnotetext{
${ }^{1}$ La población con discapacidad es diversa desde el punto de vista funcional, lo cual implica que cada tipo de discapacidad (física, visual, auditiva, cognitiva, psicosocial y de talla baja) requiere una mirada concreta sobre las barreras con las que se encuentran en su entorno. La investigación se ha centrado en aquellas personas cuyos tipos de discapacidad encuentran más dificultades en la interacción con el espacio físico en cuanto al acceso y desplazamiento independiente, que son las discapacidades con mayor prevalencia en la ciudad (ver Anexo, tabla 1).
} 
inciden desde su campo social en la planeación de la ciudad, unos desde perspectivas técnicas y políticas administrativas, y otros desde la búsqueda por hacer parte de las trasformaciones de la ciudad en su carácter de ciudadanos y desde la práctica cotidiana, pues históricamente diversas barreras o contextos no han posibilitado oportunidades para la participación en sus dinámicas, ubicándolos como ciudadanos de segunda categoría².

Es de resaltar que las perspectivas médicas e higienistas, como técnicas de planeación de la ciudad e interpretación de la discapacidad tradicionales, dejan entrever que el diseño de los espacios de ciudad obedece a estrategias foráneas, las cuales han proyectado una ciudad de espaldas a quienes la viven y la habitan, cristalizando formas excluyentes y homogeneizando las necesidades y el carácter de sus pobladores. Podemos entender que el diseño de la ciudad ha desconocido las condiciones de las personas con discapacidad, pues la planeación exclusiva de técnicos no permite, en términos de David Harvey (1979:85), diversificar las perspectivas en coherencia con la diversidad de los ciudadanos, en este caso la diversidad funcional de las personas con discapacidad.

No obstante, el reconocimiento progresivo de la discapacidad como una condición inherente a la diversidad humana y la necesidad de un abordaje integral que trascienda la mirada biológica (médica e higienista) hacia una mirada que integre lo funcional y lo social, ha permitido que desde instancias nacionales y locales se construyan políticas públicas para responder a las necesidades y garantizar los derechos de una población excluida de los procesos sociales. La ley nacional 1145 de 2007 establece un marco sistémico y territorial para implementar la Política Pública en Discapacidad, ya que a nivel municipal el Acuerdo Municipal 086 de 2009 y el decreto 221 de 2011 en el Plan Municipal de Discapacidad buscan la materialización de la política a nivel local, procesos en los cuales han participado diversos actores que incluyen organizaciones de personas con discapacidad, ONG, empresa privada y funcionarios públicos (Semillero de Investigación Observatorio de la Sociedad Civil, 2007).

En esta conjunción de acciones e interacciones se ponen de manifiesto las tensiones y acuerdos frente a la implementación de políticas que tengan como propósito la garantía de derechos a través de la superación de las diferentes barreras físicas de la ciudad. Por ello, la planeación de la ciudad, sin integrar la perspectiva de la discapacidad, puede expresar una forma de desconocimiento de su condición de ciudadanos. Este aspecto de la planificación genera un tipo de interacción particular

\footnotetext{
2 Eduardo Díaz Velásquez (2009:118), citando a T. H. Marshall, plantea que desde una perspectiva sociopolítica moderna, la ciudadanía es un "estatus que se concede a los miembros de pleno derecho de una comunidad. Sus beneficiarios son iguales en cuanto a los derechos y obligaciones que implica". No obstante, con las personas con discapacidad, como grupo minoritario, se despliegan otros debates que van más allá de los elementos identitarios y cruzan todos los componentes relacionados con el ejercicio efectivo de los derechos y la búsqueda de la participación en el sistema productivo y en su entorno físico.
} 
entre quienes planifican la ciudad y quienes desarrollan una práctica espacial a partir de un sentido de diferenciación frente a los otros, identificándose en ambos unos relatos y acciones que expresan las formas de resignificar el espacio, además de ser el ámbito donde las personas con discapacidad como ciudadanos pueden ejercer y disfrutar sus derechos en igualdad de condiciones (Borja, 2003).

Para orientar el proceso de análisis, se plantea como hipótesis general que las personas con discapacidad implementan diversas estrategias y acciones sociopolíticas para incidir sobre la planeación que la administración pública hace de los espacios de la ciudad, con el fin de superar condiciones de dominación y exclusión, y la reivindicación de su condición de ciudadanos. Así, se logró analizar los conflictos y acuerdos entre las personas con discapacidad y la administración municipal, generados a partir de la planificación y el diseño de espacios físicos públicos, con el fin de comprender las incidencias de las representaciones del espacio en la planeación de ciudad, en el contexto de su transformación urbana. Por otra parte, las preguntas por las que se orientan las reflexiones fruto de la investigación abordan cuestiones tales como: ¿Cómo y para qué inciden las personas con discapacidad en la planeación y diseño de los espacios de ciudad? ¿Qué representaciones construyen las personas con discapacidad sobre el espacio físico de la ciudad?

Al tener presente las imbricaciones propias del problema de investigación, la implementación del seguimiento a políticas, además de la garantía de una serie de derechos inscritos en un marco internacional, fue necesario generar una batería de herramientas metodológicas que se acerque al hecho de tal manera que se puedan ver las perspectivas que implican temas amplios y elementos particulares a cada uno de los actores. En esta lógica, la investigación contó con un cuerpo metodológico fundamentado en un enfoque fenomenológico crítico a través de un diseño cualitativo, con la implementación de técnicas como la observación participante no estructurada, la revisión documental y la entrevista como eje central del análisis.

De esta forma, los fenómenos fueron analizados a partir de las experiencias y su construcción en la vida cotidiana de las personas, no por la intuición del investigador, permitiendo formular el modo en que los fenómenos pueden ser universales, pues devela las estructuras de los significados de las realidades, del mundo de la vida (Rodríguez, Gil Flores, y García Jiménez, 1996), evitando toda relación estandarizada sobre variables que sistematicen los comportamientos de los individuos, lo que incluye su interacción con los otros y las interpretaciones que se construyen para y por estas interacciones (Goffman, 2006).

La revisión documental permitió dar un contexto histórico del fenómeno mismo en cada una de sus partes compositivas, lo cual fructificó en el conocimiento de elementos subyacentes, aunque no prioritarios para esta investigación: conceptualizaciones normativas, contextualización y desarrollos jurídicos, datos estadísticos, normas técnicas y arquitectónicas, entre otros. De esta forma, se identificaron los sentidos que 
se establecen en la normativización dentro del hecho social y las clasificaciones sobre la discapacidad, que construyen un marco histórico en el entendimiento de la identidad, el cuerpo y del entorno de esta población (Foucault, 2006).

Las entrevistas, técnica primaria para esta investigación, permitieron el acercamiento a los individuos y sus relatos, que es la muestra más cercana a la interpretación que hacen de una realidad múltiple y paralela a otras. De esta manera, se dio relevancia a la experiencia de las personas y su conocimiento frente al hecho, inclusive frente al tema de investigación. Además, las entrevistas ayudan a definir, desde una perspectiva Emic/etic, las categorías y subcategorías que orientan el análisis en la observación participante no estructurada, dando privilegio a través de ambas técnicas a las representaciones que los sujetos construyen del fenómeno en general ${ }^{3}$. Fueron entrevistadas 12 personas, entre líderes de organizaciones de personas con discapacidad, funcionarios públicos y personas con discapacidad sin filiación.

Estos relatos (texto, habla y acción) pueden interpretarse como formas de dominación-emancipación en el ámbito político y social, convirtiéndose en un recurso fundamental para entender la práctica espacial particular de las personas con discapacidad y su forma de ejercer la ciudadanía. Además, contienen su importancia no jerárquica, las cuales brindan la posibilidad de desentrañar distintos entramajes que permitirán develar estructuras de sentido que se puedan sistematizar y dar una perspectiva más amplia a la acción misma como expresión de un fin (Geertz, 1973).

La observación participante se planteó como el facilitador para el acercamiento a las formas en que los sujetos de la investigación llevan a cabo sus acciones cotidianas o las que están en conjunción con intereses o fines grupales. Al tener presente que la realidad no es susceptible de ser observada de forma objetiva, al ser una construcción social (colectiva), requirió entonces observar y ser observado, pues esta realidad puede ser compartida, aunque interpretada desde diversas miradas (Montañés Serrano, 2009).

El primer contexto observado fue la mesa de Accesibilidad, orientada a la implementación temática del Plan Municipal de Discapacidad. Ser parte de la Mesa no requirió del contacto con un broker o portero, pues desde el año 2012 hasta el año 2016 me desempeñé como Profesional Social del Proyecto de implementación de la Política Pública del Municipio de Medellín, con la oportunidad de participar como moderador. Esta posición permitió una recolección de información amplia y variada en torno a los temas de trabajo de la Mesa. Sin embargo, fue necesario un filtro reflexivo y consecuente con el tema y el foco de la investigación. Otro contexto de observación fue la marcha anual en conmemoración del día internacional de las personas con discapacidad, el 3 de diciembre del año 2015. Este acto anual recuerda, además, la

3 El perfil de las personas entrevistadas fue definido tras la realización de un taller sobre técnicas participativas realizado con personas con discapacidad de la ciudad, entre integrantes y no integrantes de organizaciones. Las técnicas de Sociograma y Mapa de Actores, implementadas en el taller, permitieron que los participantes plantearan qué personas eran relevantes en el tema central de la investigación y la red de contactos para entender el entramado social sobre el tema (Red CIMAS, 2015). 
promulgación de la Convención Internacional sobre los derechos de las personas con discapacidad (ONU, 2007). En ella confluyen personas de diversas organizaciones y otras sin afiliación para marchar por el centro de la ciudad exhibiendo pancartas y con proclamas acerca de su condición de personas y sujetos de derechos.

Sin ser un proceso estrictamente secuencial, se establecieron las siguientes fases de trabajo para la implementación de las técnicas y la recolección de datos: planeación del trabajo de campo, realización del trabajo de campo, sistematización de los datos, análisis de la información, construcción del marco teórico y conclusiones. Este proceso estuvo transversalizado por el acercamiento a propuestas teóricas y metodológicas que orientaron progresivamente la implementación de las técnicas como la sistematización y el análisis de la información. Finalmente, como resultado del proceso de formación y las lecturas previas, se estructuró un marco teórico que ayudó a abstraer los análisis de los resultados o datos empíricos categorizados, y a generar una triangulación por medio de la cual se construyeron las conclusiones dando respuesta a las preguntas de investigación como forma de validación de la hipótesis y del conocimiento sobre el hecho social.

\section{Las nuevas perspectivas desde los enfoques médico y biopsicosocial}

El enfoque médico ha tenido relevancia gracias a que la discapacidad se ha visualizado en la dualidad salud/enfermedad; por tal razón, desde este enfoque las personas son sujetos de intervención médica sin lugar para una perspectiva integral de su condición. El referente conceptual para este enfoque ha sido construido desde la Organización Mundial de la Salud (OMS) como la autoridad internacional destinada para ello. Desde la década de los 70 ha publicado diferentes manuales para orientar la intervención sobre la discapacidad, planteando inicialmente la discapacidad como un asunto de salud pública que requería tratamiento o intervención médica. Así, la discapacidad era vista como un fenómeno causal de un proceso lineal que iniciaba con una enfermedad, pasaba a una deficiencia y posteriormente de discapacidad a minusvalía (Egea García y Sarabia Sánchez).

Desde el desarrollo del enfoque social, dada una serie de discusiones conceptuales e incidencias políticas y sociales, y en vista de la utilización negativa de los términos de su clasificación, en 2001 la OMS presenta la Clasificación Internacional del Funcionamiento, la Discapacidad y la Salud -CIF- (OMS, 2001) que aborda la discapacidad desde una perspectiva biopsicosocial, ampliando la comprensión del fenómeno no solo desde sus consecuencias en el ámbito médico, sino desde la interacción entre diferentes sistemas y contextos sociales, políticos y económicos. Así, la discapacidad ya no es vista desde esferas que la intervienen como una enfermedad, sino como inter y correlación de diversos factores como la deficiencia fisiológica, la interacción social y los elementos socioambientales que no le permiten participar en la vida social (Egea García y Sarabia Sánchez, s.f). 
En lo local, los avances en las perspectivas se materializaron en el diseño e implementación de políticas de carácter público para establecer una plataforma de acción a favor de los derechos de las personas con discapacidad. Desde la ley nacional 1145 de 2007, la ciudad de Medellín dio respuesta a esta ley con la implementación de la Política Pública en Discapacidad a través de un Plan Municipal de Discapacidad, la cual fue construida con la participación en talleres, foros y conferencias de diferentes actores implicados en el ámbito de la discapacidad.

Así, se ha buscado resignificar la discapacidad como un hecho multisectorial que no es suficiente abarcar solo biológicamente. Pero no es la política pública la que llama a esta búsqueda, han sido los mismos actores involucrados, dando sentidos diversos a lo que es la discapacidad, desde sus experiencias y quehaceres. Por ello, desde 1978 la OMS viene desarrollando la estrategia de empoderamiento denominada Rehabilitación Basada en Comunidad (RBC), planteada como una estrategia para la superación de la pobreza y la generación de procesos autónomos desde las comunidades y las organizaciones, para que las mismas personas con discapacidad identifiquen sus necesidades en diferentes campos y así adelantar acciones que propendan al diálogo intersectorial como una forma de desarrollo social y comunitario en un proceso continuo de defensa y garantía de los derechos (OMS, 2004).

Esta propuesta se vincula con los modelos extendidos y holísticos que buscan superar no el enfoque biomédico, sino el abordaje unívoco de la discapacidad; el biopsicosocial, desde el cual se genera una visión integral de la discapacidad como un fenómeno presente en el sujeto desde sus diferentes dimensiones relacionales (Egea García y Sarabia Sánchez). Por otro lado está el modelo ecológico, que desde la propuesta de Bronfenbrenner (1987: 12) pone a la discapacidad como institución social que transversaliza todas las dimensiones en las que la persona con discapacidad interactúa a través de sus distintos roles sociales y sobre las cuales puede intervenirse para adaptarlas a las condiciones mismas del sujeto en búsqueda de su inclusión y participación.

\section{Resultados: vivir con discapacidad en la ciudad}

A través de los relatos desarrollados en las entrevistas, las personas con discapacidad expresaron las condiciones en las que deben interactuar en medio de las barreras físicas y actitudinales con las que se encuentran en la ciudad. Estas barreras, desde la perspectiva de algunos entrevistados, se convierten en la causa para que las personas con discapacidad se confinen en sus casas generando dos situaciones: que no sean visibilizadas en los diferentes espacios $y$, por consecuencia, los imaginarios que se tienen de estas no puedan ser transformados, pues se replica la idea de que no "deben" ni "pueden" salir de sus casas; una forma de exclusión y discriminación soterradas. 
Es así como la Accesibilidad, más que un concepto elaborado técnica $\mathrm{y} / \mathrm{o}$ jurídicamente, es una forma de exigir y responder a la inclusión con acciones que garanticen la igualdad de oportunidades para la población diversa que habita y vive la ciudad; se despliega como estrategia para ratificar que hay personas que deben ser reconocidas en su condición de ciudadanos, para lo cual ha de trascenderse su condición biológica o mental y, por ende, la planeación y el diseño de espacios físicos deben tener presente estas condiciones ${ }^{4}$. En esta perspectiva puede encontrarse además que las barreras físicas también se producen por otro tipo de barreras quizás más complejas como son las actitudinales, las cuales se ubican como barreras que se construyen culturalmente y que se superponen al conocimiento técnico de los expertos en diseño y planeación haciendo parte de su visión de las realidades.

Así, la mirada a las categorías Accesibilidad y barreras físicas permite dilucidar algunas referencias directas a la importancia que los líderes con discapacidad dan a las barreras como elementos que no posibilitan la participación social y política sin ningún tipo de discriminación de las personas con discapacidad, es decir, elementos que imposibilitan la ciudadanía y que no generan inclusión porque son el resultado de prácticas espaciales de personas y organizaciones -planificadores y otros ciudadanos- que desconocen la discapacidad como parte de la diversidad humana. A esto, uno de los líderes de personas con discapacidad, además funcionario contratista de la administración municipal, plantea que:

tampoco es hacerle la ciudad de los ciegos porque de pronto... o la ciudad de la gente con discapacidad física. ¡No! O la ciudad de los sordos. ¡No! Es ¿cómo hacemos ese cóctel perfecto, esa mezcla perfecta que no se note tanto para nadie y que le haga la vida cómoda a todo el mundo? Una tarea un poco difícil, pero ese es el reto y el desafío (integrante de Fuerza Incluyente y funcionario público, persona con discapacidad visual, 2015).

Estas barreras solo son catalogadas como tal una vez que los sujetos desarrollan acciones que pueden ser cotidianas o no, pero que son acciones que están determinadas en la relación del individuo con el espacio, no solo con lo que este representa en la lógica de su construcción o configuración física, sino su posición en las

\footnotetext{
${ }^{4}$ Ante la amplitud del concepto se plantea una perspectiva puntual para este artículo. Por un lado, David Harvey (1979) da cuenta de la accesibilidad como parte de la estructura urbana, un rasgo que permite el acceso a servicios desde el punto de vista de las familias consumidoras de servicios. Esta se logra cuando las familias pagan el precio requerido para el acceso a estos servicios dadas las distancias y el tiempo para acceder a ellos. Para Iwarsson y Stahl (2003), es un concepto, además de relativo, amplio, donde se ponen con relación a la funcionalidad de las personas y el diseño del entorno. En este artículo veremos cómo este concepto toma sus matices según el contexto analizado desde la óptica de las personas con discapacidad, pasando de ser el resultado de miradas técnicas, jurídicas y teóricas a un dispositivo para la plantear la necesidad de incluir las perspectivas de la discapacidad en la estructura urbana y asumir el reto de nuevas estrategias para avanzar a la inclusión.
} 
jerarquías que tengan estos lugares, cuando son vividos, percibidos y/o concebidos, particularidad presente en la comprensión trialéctica del espacio de Lefebvre (2013). No obstante su jerarquización, la legislacion nacional, especificamente la ley estatutaria 1618 de 2013, en su articulo 14, vincula a todos los entes territoriales a garantizar el acceso de las personas con discapacidad tanto a servicios como espacios en igualdad de condiones, con el fin de garantizar la igualdad material y el fomento de una vida autónoma e independiente (Ley estatutaria 1618, 2013). Esta igualdad de condiciones significa establecer acciones y mecanismos para que todos los ciudadanos tengan garantizado el goce efectivo de derechos.

Con relación a su vida cotidiana como categoría de análisis, se hallan elementos por medio de los cuales se presume la percepción de lo que significa vivir con discapacidad. El asistencialismo emerge en las narraciones como una actitud que denota maneras de relacionarse con la discapacidad como fenómeno en general y de características generalizadas a toda esta población, pero, a su vez, una forma de generar acciones en el contacto cotidiano con las personas con discapacidad, es decir, como fundamento de la interacción entre sujetos e instituciones. Estas actitudes son entendidas como una forma de asimilar la imposibilidad de llevar una vida "normal", que desde la mirada de las personas "normales" limita la capacidad de valerse por sí mismo. Así, las personas con discapacidad son vistas como personas "anormales" y dependientes, que requieren la asistencia de otros para realizar actividades de la vida diaria, pues no pueden llevar una vida "normal". Si bien las personas con discapacidad reconocen que aun requieren la ayuda o acompañamiento de otros -principalmente las personas con discapacidades más graves-, lo que buscan es la autonomía y la independencia que les permitan llevar su vida con dignidad, la vida tranquila que lleva una persona sin discapacidad. Esta forma de llevar la vida también se convierte en una forma de cambiar este imaginario con acciones prácticas o cotidianas.

Uno de los relatos de los líderes de organizaciones de personas con discapacidad entrevistado reúne los elementos expuestos bajo esta categoría:

El bastón me salvó... seguí caminando para cruzar la avenida. ¡Oh!, casi no la cruzo. Un señor me ayudó y casi nos coge una moto porque el que venía bajando... el bus... pitó para que pasáramos y el que venía en sentido contrario, absolutamente no pitó... Él ya me colocó en todo el bordito del puente para llegar hasta la estación Tricentenario (Metro)... Subí las escalas, me desplacé en el Metro hasta la estación y me pasó una cosa muy curiosa en la plataforma. Yo me bajé del Metro solo, el auxiliar de la estación no me reportó. No estaba, pero entonces yo empecé a caminar y ¡oh, sorpresa! Me encontré que en la 
plataforma ya pusieron la línea táctil ${ }^{5}$. Y me fui viniendo, viniendo, cuando el auxiliar me encontró ya llegando a las escalitas. Dizque: “¿ué hubo?" y yo: "¿Qué hubo?". "No me lo reportaron". Yo: "Tranquilo que ya. Aquí está la línea y yo me vine muy relajado". Yo venía todo relajado por toda la plataforma. ¡Qué ayuda tan buena fue esa! (líder del Comité Comunal de Inclusión, persona con discapacidad visual, 2015).

Este relato manifiesta la angustia que supone salir a la calle, pues el espacio no es tan conocido y controlado como la casa, ya que en el espacio público puede encontrarse con muchas barreras que lo ponen en peligro; en otras palabras, para él mismo el espacio público no está hecho para las personas con discapacidad; de igual manera lo determina el auxiliar de policía, quien percibe que por ser una persona con discapacidad no tiene otra forma de orientarse en el espacio si no es con la asistencia de otros. No obstante, su expresión de entusiasmo surge cuando relata lo acaecido en su desplazamiento por las estaciones del Metro, donde la línea táctil hace la diferencia, pues le permite independencia y como forma de transformar imaginarios. Así, para las personas con discapacidad, la asistencia es necesaria en ciertos contextos, pero es mejor la accesibilidad que apunta al reconocimiento de su condición de ciudadanos con derecho al desplazamiento en la ciudad.

En el ámbito de las acciones colectivas se agrupan inclusive las acciones cotidianas que tienen una carga de acción política en la medida en que, en cada oportunidad que se presenta en la interacción con otras personas, es una oportunidad para dar a entender que como ciudadanos tienen el mismo derecho a acceder y disfrutar de los espacios en tanto públicos, aspecto desarrollado ya, pero como colectividad logran movilizar otras acciones que conjuguen estas miradas particularizadas y compartidas. Tal es el ejemplo de otra persona con discapacidad física, líder de la Mesa de Discapacidad de la comuna 6 de la ciudad, quien propone un trabajo de cambio de imaginario desde las familias de las personas con discapacidad, pues desde su experiencia se encuentra con los miedos de las familias a la posibilidad de que sus familiares participen activamente como ciudadanos de la vida social y política, lo cual implica trascender las barreras de la vida privada (la casa) y exponerse a los riesgos del entorno en la vida pública:

la ciudad ha avanzado en eso y creo que debe seguir trabajando la pedagogía sobre eso; no solo con la persona con discapacidad, sino con el cuidador y con su entorno, y es importante también que desde ustedes, que son sociólogos, trabajen y lleven siempre un puntico en todo lo que ustedes hacen sobre líderes comunales, sobre organizaciones. Mire, trabajen esto; mire, tengan en cuenta la inclusión, no solo para

\footnotetext{
${ }^{5}$ Su nombre técnico es señal táctil para superficie peatonal STSP (Indicador táctil de superficie de caminado). Y es definida por la Norma Técnica Colombiana 5610 (NTC 5610) "como Superficie normalizada de caminado que sirve de guía para facilitar el desplazamiento de personas con limitación visual" (ICONTEC, 2008: 2).
} 
nosotros, sino para todos; mire, tengan en cuenta el diseño universal, y eso ayudará mucho a seguir mejorando (persona con discapacidad física y líder de la Mesa de Discapacidad, comuna 6, Medellín, 2015).

Es así como quienes asumen el reto de vivir su vida en el ámbito de lo público se orientan al desarrollo de actividades de incidencia en aquellas instancias que se vienen desplegando para ello. Las mesas temáticas del Plan Municipal de Discapacidad y los Comités Comunales y Corregimentales de Inclusión $^{6}$ son aquellas instancias importantes para la ciudad como escenario de discusión y acciones conjuntas entre actores con y sin discapacidad, a favor de la accesibilidad como derecho y forma de reivindicación del mismo.

En el espacio de la mesa, la normatividad y las obligaciones institucionales fueron los temas que han generado diversas tensiones en la interacción entre personas con discapacidad y funcionarios, pues las discusiones se centraron en el desplazamiento de responsabilidades en otros actores, permitiendo identificar allí que en la mesa el conjunto de elementos que movilizaron la participación están constituidos por una serie de cuestionamientos sobre lo que pasa en la ciudad en el tema de la accesibilidad. Además, surgen interrogantes sobre la efectividad de la mesa por sí misma, ya que los reclamos mutuos sobre responsabilidades dejan en evidencia la poca o baja capacidad de incidencia de la mesa a pesar de la presencia de diversos actores.

Aunque se avanzó en la implementación de otras estrategias o acciones delegadas, el tema de las responsabilidades institucionales no desapareció, convirtiéndose la mesa en una instancia de tensiones sobre temas particulares, donde los representantes de las organizaciones manifestaron su inconformidad con lo que para ellos son omisiones de la administración municipal frente a "deudas históricas" con una población que aún es discriminada. Ante estos reclamos, los funcionarios fueron comprensivos, viéndose advocados a precisar las limitaciones que tienen para tomar decisiones, pues la norma que los rige y sus funciones misionales están supeditadas a instancias de orden directivo superior, a las que proponen llegar para lograr los avances necesarios en el tema con la ejecución de acciones conjuntas.

\footnotetext{
${ }^{6}$ Instancias de participación y discusión de temas en relación con la discapacidad. El proceso de observación desarrollado en la investigación tuvo como uno de sus focos la denominada Mesa de Accesibilidad desde el año 2012 al año 2015, donde se abordan temas relacionados con la accesibilidad a la infraestructura, el transporte y las comunicaciones. En esta participan el Departamento Administrativo de Planeación, encargado del seguimiento de todas las políticas públicas poblacionales, la implementación del Plan de Desarrollo y el Plan de Ordenamiento Territorial; la Secretaría de Movilidad y Transporte, encargada de planificar, regular y controlar todo lo relacionado con la circulación peatonal y vehicular; la Unidad de Discapacidad, encargada de direccionar diversas acciones de intervención sobre la población con discapacidad; Metro y Metroplús, dos empresas encargadas de ejecutar y coordinar el transporte masivo de pasajeros en todo el Área Metropolitana. Además, participan organizaciones de personas con discapacidad tales como Fuerza Incluyente, organización dedicada a la movilización de sus integrantes con actividades de visibilización; la Red de Discapacidad REDDIS, nodo Medellín. También hacen presencia actores de carácter comunitario como los Comités Comunales y Corregimentales de Inclusión (CCCl), creados a partir de la implementación de la política pública.
} 
Ente las exigencias de las personas con discapacidad, los funcionarios presentes en la mesa dispusieron su capacidad misional y su nivel de decisión para capacitar a los integrantes en temas de carácter normativo y técnico, con el propósito de orientar acciones posteriores que lleguen a quienes tienen la obligación administrativa de dar respuesta. Por esta razón, desde el año 2014 se establecieron encuentros para abordar aspectos como: estrategias del transporte masivo para la garantía del acceso y la accesibilidad de todos los usuarios al sistema, las Normas Técnicas Colombianas (NTC), entre ellas, la NTC 4145 y la NTC 6047 sobre Accesibilidad al medio físico y espacios de servicio al ciudadano en la administración pública. En estos abordajes es característico de las personas con discapacidad el uso de la Convención Internacional sobre los Derechos de las Personas con Discapacidad como herramienta para argumentar la necesidad de garantizar el derecho a la accesibilidad en los espacios públicos por medio del diseño universal (ONU, 2007).

Si bien para el año 2015 se habló de la participación de las personas con discapacidad en los diseños y construcciones arquitectónicas como una forma de inclusión en la planeación, nunca se plantearon las estrategias a largo plazo para ello. Los funcionarios fueron cautelosos frente al tema ya que, según ellos, se requiere una capacitación en elementos técnicos, lo cual es complejo de acuerdo al nivel de conocimientos de las personas y los costos que ello acarrea. No obstante, las personas con discapacidad aceptaron que una comisión de trabajo de la mesa construyera lo que se denominó Instrumento de Verificación de Accesibilidad (IVACCES), el cual en su primer capítulo abordaría la Accesibilidad al medio físico (ver Anexo, imagen 1). Esta iniciativa, buscando la inclusión tanto en el diseño como en el desarrollo de un elemento ampliamente técnico, despertó muchas expectativas entre las organizaciones. No obstante, la coyuntura electoral y el cambio de administración entre el año 2015 y el año 2016 frenaron el proceso, pues tuvieron que centrarse en dar a conocer el trabajo y los logros de la mesa con los nuevos funcionarios directivos para que las acciones tuvieran continuidad.

Los funcionarios han encontrado entonces que existen imaginarios que condicionan la respuesta de otros funcionarios y de ciudadanos frente a la discapacidad, los cuales deben ser intervenidos. Pero para ello se requiere incidir a los funcionarios de niveles directivos de la administración municipal y sobre sectores que tienen alta influencia económica y política en la ciudad. De esta forma, los integrantes de la mesa han visualizado algunas acciones a largo y mediano plazo en dos vías: la primera, propender por la capacitación y formación de otros funcionarios en la administración municipal en temas de inclusión y discapacidad, tomando partida de la posesión de un nuevo alcalde con un equipo nuevo de funcionarios y asesores para el periodo 20162019. La segunda, realizar un trabajo de relacionamiento con otros sectores públicos y privados que aún no se suman de forma directa al tema de la accesibilidad y la 
discapacidad, actores que fueron identificados en un ejercicio de mapa de actores y Sociograma, asesorado por la Unidad de Discapacidad (ver Anexo, imagen 2).

\section{Análisis y discusiones centrales}

Al tener un gran conjunto de información -datos cuantitativos y cualitativos- sobre la discapacidad, tanto local como nacional e internacional, es necesario filtrarla para entenderla en el contexto en que se delimita esta investigación. De esta, a su vez, tenemos unas amplias interpretaciones según la utilidad, la perspectiva individual o grupal, e inclusive institucional. Por ello, se entiende este mar de información como una lucha que ha ido definiendo desde la comprensión de la discapacidad como un aspecto de la diversidad psicobiológica del ser humano interpretada e intervenida por la técnica médica, la que al mismo tiempo determinó durante mucho tiempo el carácter de su sentido social y delimitó además de la interpretación, las posibilidades de acción de los sujetos. No sobra resaltar que esas interpretaciones de lo que es la discapacidad perviven y persisten en distintos contextos -escenarios en términos de Goffman-.

Para el caso concreto de Medellín, estas disputas por la identidad y el reconocimiento de la discapacidad se expresan en asuntos de carácter administrativo como la implementación de mediciones y estudios, así como de la generación de lenguajes "correctos" que impliquen un cambio de imaginarios en todos los ciudadanos. Estas acciones por el reconocimiento y la interpretación de la propia identidad en la ciudad conllevan en sí la búsqueda de respuestas de carácter político y social a un hecho como la discapacidad que se manifiesta, con las acciones conjuntas, individuales cotidianas o no de las personas con discapacidad, como un hecho social que es transversal en muchos escenarios sociales.

Tenemos, entonces, un conjunto de miradas que, aunque amplias y diversas, nos derivan hacia la comprensión de asuntos referidos a la planeación urbana y la participación, y cómo estas mantienen una causa-efecto-causa en la configuración de sus formas. En primer lugar, encontramos estructuras normativas y disciplinarias que se conjugan para dar un marco de referencia en la interacción entre sujetos y ciudad. Desde las perspectivas históricas para comprender la discapacidad, hasta las propuestas más actuales que determinan las miradas integrales sobre la discapacidad como un asunto social que no se desliga de los aspectos biológicos, los integra. Estas propuestas, aunque tienen una meta orientada hacia la integralidad en el manejo del tema, también hacen parte de los marcos generados desde la ciencia para determinar las formas en que el Estado y los ciudadanos deben actuar frente al hecho social, incluso las mismas personas con discapacidad.

Por ello, existen por parte de las personas con discapacidad unas apuestas por la transformación de imaginarios que se fundamentan en las situaciones de la vida cotidiana, aquellas donde el sujeto entra en interacción con todos los elementos de su entorno, lo cual incluye a los otros y el conjunto de interpretaciones de los otros como 
parte de la conformación de un marco de referencia para interactuar; pues si bien hay una serie de actuaciones que busca como medio o dispositivo de incidencia los de carácter colectivo -acciones pedagógicas, participación en políticas públicas y en otras instancias de participación de la ciudad-, manifiestan que la transformación de imaginarios también depende de la creación de posibilidades para su autonomía y un trato más equitativo, pero al mismo tiempo un desarrollo de actividades cotidianas más ajustado a sus necesidades particulares y no solo a la intervención del aspecto físico de la ciudad (Foucault, 2006).

Por otro lado, se identifican las estrategias estatales para el abordaje del fenómeno, fundamentadas en propuestas globales que plantean los elementos necesarios para comprenderlo e intervenirlo, propuestas como la Rehabilitación Basada en Comunidad, la normatividad tanto jurídica como técnica, internacional y local, entre otros. Si bien estas estrategias son acogidas por los movimientos asociativos de personas con discapacidad como estrategias que otorgan posibilidades de comprender sus condiciones y poder ampliar las propuestas de intervención a través de sus acciones, aquí aparece entonces la tensión con los entes administrativos porque las estrategias no son implementadas de forma efectiva y porque estas, a su vez, solo discurren en diálogos y decisiones que hacen más lentas las acciones de la administración para garantizar la inclusión de la población con discapacidad de la ciudad.

Al mismo tiempo, encontramos en este abordaje de elementos normativos y jurídicos, en cuanto a la accesibilidad e inclusive a la construcción social de la discapacidad, una suerte de flexibilización que permite que las personas con discapacidad puedan proponer las formas de trascender los procesos de "normalización médica" y normativización del tema, y generen sus propias estrategias integradoras, es decir, la combinación de diversas perspectivas para la transformación de las miradas hegemónicas científicas o políticas (Foucault, 2006), trabajo bastante complejo por la amplitud de su campo de acción y lo que representa ser ciudadanos que exigen ser tratados como tales.

Así, las personas con discapacidad no se detienen en su esfuerzo por incidir sobre los procesos de inclusión desde su participación en instancias institucionales intersectoriales, como las mesas del Plan Municipal de Discapacidad o los $\mathrm{CCCl}$, por un lado, y por otro lado, la vida cotidiana surge como un campo de acción efectivo para la transformación de imaginarios y el reconocimiento de las capacidades de las personas con discapacidad, las cuales se manifiestan tanto en sus luchas por una ciudad incluyente a través del diseño de espacios accesibles como en el disfrute de los espacios de ciudad una vez que estos se adaptan a las condiciones de los ciudadanos que los usan.

Borja pone como fundamento de la relación entre la ciencia y lo normativo en el tema de la planeación de la ciudad, un proceso que conlleva, primero, una etapa cultural de construcción de los valores característicos o constitutivos de los derechos en el sistema 
urbano, y segundo, un proceso de movilización ciudadana que promueva la inclusión de estos derechos en marcos normativos territoriales, que a su vez impulsen procedimientos de participación en el desarrollo de políticas que permitan la garantía de tales derechos (Borja, 2003)

Estos discursos demuestran que ya no se ven a sí mismos y no quieren ser vistos como pacientes o personas enfermas, determinación que se rompe con la promoción de su ciudadanía y su actuación como tales -sujetos de derechos- que están activos en la vida social y que buscan ser reconocidos como minoría de ciudadanos con criterios, opiniones y perspectivas de sus derechos. Esta dimensión es la que puede permitir entender que la estructura física de la ciudad tiene un sentido complejo que supera la determinación técnica o lo estético-artístico y que tiene un sentido que se transforma históricamente desde la inclusión de nuevos actores en su desarrollo (Borja, 2003: 2526). Este giro se logra no solo por el conjunto de normativas y convenciones internacionales, sino por la persistencia y fuerza del sentido identitario colectivo de las organizaciones y personas con discapacidad.

A partir de esta lectura crítica que se expresa en los testimonios, en las posiciones de la mesa de discapacidad y en las acciones colectivas en el espacio público, se puede identificar cómo el espacio social connota una dimensión intersubjetiva y política a la vez; las personas con discapacidad subrayan la integralidad de la vida privada y la vida pública, ya que para ellas, con sus condiciones y capacidades, están diariamente experimentando ambos escenarios $y$, en especial, resaltan los vacíos para que ambos escenarios se integren. Entonces, el diseño de espacios accesibles es un elemento que, desde estas perspectivas, integra distintas apreciaciones sobre lo que debe ser un espacio para todas las personas; además, pone en cuestión las habilidades geográficas y técnicas de quienes planifican y diseñan, desconociendo la forma en que las personas son influidas por el espacio y su finalidad pero, sobre todo, cómo ellas mismas buscan reconfigurar lo que denomina Harvey la forma espacial a través de los procesos sociales, teniendo presente que ninguno de los dos está supeditado al otro y por ello es necesario generar un marco teórico que ayude a entender cómo emergen las formas urbanas y espaciales, además, cómo están ligadas entre sí en su proceso (Harvey, 1979: 19-20).

\section{Conclusiones}

Al retomar la hipótesis de esta investigación y las preguntas que la orientaron, podemos establecer algunas conclusiones en tres aspectos: en el primero, se considera que las personas con discapacidad están incidiendo en la forma cómo se interpreta la discapacidad, ya que más allá de ser una condición biológica, toma su sentido como una construcción social, cuyos componentes se alimentan del contexto histórico y espacial en los que operan las diversas visiones sobre la organización de la vida social. En segundo lugar, la planeación y diseño de espacios físicos en Medellín se 
fundamenta en lógicas que están inscritas en un orden global que pasan, además, a los contextos locales tras el cumplimiento de una serie de normas que rige sobre el país. Por ende, la planeación y el diseño en su fundamentación no contemplan para las personas con discapacidad las realidades vividas en su cotidianidad y, en consecuencia, se proyectan desde sus organizaciones las formas o acciones pertinentes para lograr incidir, aunque sea en un contexto más cercano, sobre la configuración de los espacios.

Además, desde la perspectiva de las acciones y las condiciones de las personas con discapacidad, los espacios adquieren sentidos diversos no solo a través de la exigencia del cumplimiento de la norma, sino a través de la interacción cotidiana con ellos y con esos otros que también los viven y los habitan. De ahí que la exigencia de espacios accesibles esté cruzada por la implementación de la norma y la trascendencia de la misma por medio de la participación de las personas en su diseño, no como una simple forma de modificación o transformación de los mismos, sino como la oportunidad para visibilizar a una población que ha estado relegada a un segundo plano en lo que se define como planeación participativa en los entes municipales. Es así como lo expresa una de las personas entrevistadas al comentar:

conozco mucha gente de ese lado, por ejemplo comuna 5, comuna 6, que yo sé que les va a beneficiar ese transporte, hablé con ellos y algo ha de servir. Unas 15 ó 20 firmas de ese lado, con una carta y una foto soportando lo que estamos diciendo, yo creo que de algo ha de servir (persona con discapacidad física, 2015).

Este cambio en la mirada sobre la discapacidad, expresada constantemente por las personas con discapacidad en el contexto de Medellín, consiste en la transformación de la lógica que deviene de la interpretación de la vida en la ciudad, mediada por la mirada higienista, la cual marcaba las pautas para la configuración de los espacios y la determinación de las formas en que los ciudadanos interactúan con estos, y parte de la búsqueda de la idea de progreso a través del urbanismo y la arquitectura (González Escobar, 2007: 150). Esta transformación surge además como una estrategia que deriva no solo por un contexto internacional, sino que toma sentido en lo local a través de la intención propia de ser parte de los procesos sociales dentro de la ciudad.

Documentos como la Convención Internacional sobre los Derechos de las Personas con Discapacidad, la Clasificación Internacional del Funcionamiento de la OMS y algunas normas locales que establecen una Política Pública para el país, se convierten en herramientas jurídicas de primera mano para interpelar las visiones tradicionales que la ciencia médica ha dispuesto como formas de interpretar el mundo. A pesar de su bajo conocimiento técnico en normas o epistemología, acuden a la experiencia personal y a la baja complejidad de la Convención para procurar transformar la visión 
de funcionarios y demás ciudadanos sobre sus condiciones tanto físicas y mentales como económicas y sociales.

David Harvey pone de manifiesto que en la planeación de la ciudad los técnicos se juegan su capacidad conceptual y su creatividad al procurar interpretar las formas espaciales indicadas y que estén en correspondencia con las orientaciones globales del desarrollo, la distribución de los ingresos y las particularidades presentes en la ciudad y sus pobladores (Harvey, 1979: 19). Para Foucault, en cambio, la dicotomía entre la formación del Medio como el conjunto de elementos físicos y las formaciones sociales es la estrategia inadvertida para analizar y comprender las formas de interacción con los otros, o más bien de conocer cómo se tejen los vínculos en las interacciones de los sujetos y el papel que la configuración del espacio físico cumple en las formaciones sociales y viceversa.

En contraste, en los relatos de las personas con discapacidad se encuentra cómo los dispositivos que ahora se ciñen como elementos fundamentales para dar sentido a la discapacidad y su contexto, tales como los normativos o jurídicos y de las ciencias, son funcionales a su búsqueda de transformación de la perspectiva tradicional que interpreta la discapacidad como una condición exclusivamente médica o inclusive de salud pública, ya que se ejerce un control sobre ellos que no les permite demostrar que, con ajustes sobre los entornos y sobre la misma actitud de las personas, pueden hacer parte de los procesos productivos en una sociedad, dejando de ser pacientes o enfermos que requieren ser "curados", normalizados en términos de Foucault (2006) a ciudadanos que presentan otras necesidades específicas, que no solo tienen que ver con su condición, sino con las condiciones materiales en las que se desarrollan y en las que viven.

Aunque podemos identificar en la discapacidad elementos propios de una clasificación de los individuos por su condición, hemos de centrarnos primero en la interpretación que ellos consideran debe hacerse de la discapacidad, como una apuesta por orientar las formas de intervenir el espacio físico, público o privado. Si bien esta discusión tiene un componente primordialmente subjetivo, está conectado con otros elementos que podemos denominar dispositivos que suelen ser de carácter formal por su origen. En primer lugar, si la discapacidad es entendida como parte de la diversidad humana, de ser distinto con los mismos derechos; por otro lado, esto equivale entonces a que los planificadores se verán abocados a pensar cómo planear según las condiciones sociales con las que estas se familiarizan en el contexto de la discapacidad, correspondiendo además a lógicas superiores que establecen parámetros generales desde lo técnico hasta lo político. A esto apunta el comentario de uno de los funcionarios entrevistados cuando dice:

no podríamos esperar que, caso concreto urbanizadores y constructores, de buena manera, por su propia cuenta, resolvieran si hacer construcciones 
accesibles, construcciones con el cumplimiento de todas las normas, porque tienen argumentos de peso y que tienen que ver con los costos. Están equivocados, por supuesto, pero ellos lo argumentan desde el punto de vista de los costos. (...) es necesaria la pedagogía, la formación de conciencia social, pero sobre todo la sanción por el incumplimiento de normas que ya existen (funcionario público, Alcaldía de Medellín, 2015).

De modo que, en la perspectiva de las personas con discapacidad, generar espacios accesibles no es solo una labor encaminada a dilucidar formas arquitectónicas novedosas y funcionales que cumplan con la normatividad, pues este proceso conlleva entender los sentidos de los espacios, es decir, mediar entre los empujes de las lógicas globales en los que se inscribe la ciudad, la red de producción a la que se ajusta como espacio que se concibe y que puede ser calculado y objetivado. A esto se le suman las dinámicas más particulares de la cotidianidad de los sujetos, ese espacio percibido a través de la interacción de los individuos, quienes traen consigo una carga histórica y simbólica del espacio de su identidad misma, un estigma que buscan transgredir, pero que a su vez aplican como dispositivo de resistencia y transformación de su propia historia y de los marcos de referencia en la interacción con los otros y el entorno a través del lenguaje y las acciones. (Goffman, 2006; Lefebvre, 2013).

Si bien hay conocimiento de la necesidad de implementar la normatividad por parte de distintos entes públicos en el tema de la planeación y diseño de espacios accesibles, las valoraciones sobre el tema están sustentadas sobre las vivencias en la cotidianidad, sobre los vínculos inscritos en ese orden próximo, el cual implica, en contraste con el planteamiento de Harvey, no solo los vínculos entre distintos grupos, sino además cómo se logran estos vínculos cuando se habla de la accesibilidad a los espacios como un derecho de todo ciudadano. Es en este Orden en el que las estrategias que los líderes con discapacidades entrevistados se proyectan con el fin de lograr incidencia en el tema, se sustentan sobre todo en movilizar a los ciudadanos a entender que la accesibilidad no es un asunto de discapacidad simplemente, sino un asunto de inclusión para todos.

Aunque las personas con discapacidad precisan de entender la ciudad en un conjunto, de acercarse a las técnicas planificadoras de la misma, su percepción sigue siendo una combinación de experticias personales y conocimiento jurídico para hacer exigencia de sus derechos; exigencias que van desde la interacción con y en el espacio hasta la búsqueda de instancias donde puedan aportar sus visiones y procurar transformar la de otros que pueden acercarlos a niveles superiores en la toma de decisiones. Pero cabe preguntar cómo operan los dispositivos de control una vez que se convierten en herramientas o estrategias de las organizaciones para jugar a favor de su inclusión en las lógicas de la planeación, además cómo a su vez estos dispositivos pueden llegar a ser cuestionados por ellos mismos. 
Existe una flexibilidad de las organizaciones y de las personas con discapacidad para dar a entender que la configuración de los espacios está determinada por un número considerable de elementos que pueden ir y venir según las necesidades y el contexto mismo de la interacción con funcionarios, una suerte de estrategia maleable que tiene como fin indagar, verificar o configurar los métodos apropiados de planificar y diseñar el entorno físico adecuado para su propio disfrute de la ciudad; estrategia en la cual suman el plus de la universalidad en la búsqueda de evitar la segregación involucrando a todos los ciudadanos en sus proceso de inclusión. Muestra de ello son los recorridos en campo para verificar la accesibilidad y las posibilidades de diseñar de forma que se garantice como derecho (ver Anexo, imagen 2).

Jordi Borja (2003: 220-222) considera que ante el crecimiento de las ciudades y la generación de espacios excluyentes con grupos marginados, los planificadores urbanos pueden contener la oleada de segregación a la que se ven avocadas las ciudades actuales por medio de la integración a todo proyecto urbano de tres objetivos integradores:

1. La creación de espacios que refuercen identidades

2. El reconocimiento de la diferencias

3. La existencia de momentos y lugares de expresión universalistas.

Estos tres objetivos plantean entonces la necesidad de llamar a la participación en la planeación a los grupos marginados; no obstante, en el caso de Medellín se han presentado diversas dinámicas frente a la participación. Por un lado, la construcción de una política poblacional del orden nacional fue el resultado de diversas incidencias ante el gobierno de organizaciones de personas con discapacidad y las directrices internacionales; por otro lado, ha sido la administración municipal, por medio de contratistas, quien ha dado aplicabilidad a esta política con la conformación de mesas de trabajo y comités de carácter comunal para la dinamización de esta política en los distintos niveles territoriales.

De este contexto pueden surgir muchas aristas analíticas con respecto a la autonomía de las personas con discapacidad para organizarse y actuar; sin embargo, es importante señalar que la relación Estado-organizaciones de personas con discapacidad, en el contexto de participación de la Política Pública en Discapacidad, no pasa de algunas tensiones con respecto a la efectividad de la implementación de la política y las acciones encaminadas para responder a las necesidades, asunto que es superado una vez que los actores son convencidos en la interacción de que todos son responsables de la efectividad y el avance de los planes y programas derivados de tal política, y que es más efectiva la participación constante y continuada que la presión o acciones directas en la cotidianidad. 
Esta investigación abarca asuntos muy concretos en relación con la planeación de la ciudad; no obstante, ha sido necesaria la revisión de elementos más generales vinculados al foco mismo. Por un lado, tenemos elementos propios de la identidad de ciudadanos que hasta hace poco fueron considerados "ciudadanos de segunda categoría", y por otro, aspectos relacionados con el diseño arquitectónico y la planeación de todo el territorio, asuntos que están interconectados con lógicas globales. Por ello, los autores seleccionados para el marco teórico son de corrientes diversas, entre los cuales se buscaron puntos de encuentro, desencuentro y de inflexión para el análisis del hecho investigado.

Por último, vale la pena enumerar que se derivan de este ejercicio otros temas de investigación. Uno de ellos es la definición de una identidad de las personas con discapacidad en la ciudad de Medellín, que incluya una mirada historiográfica de esta en los periodos de crecimiento demográfico. Otro tema de investigación pertinente en los procesos de implementación de políticas es la mirada hacia la interacción de ciudadanos con discapacidad, administradores o funcionarios públicos como una forma de interrelación social que puede denotar dispositivos de control o formas de incidencias y resistencias. Como reto adicional está el de promover desde la academia estrategias que ejecuten la investigación de carácter participativo como forma investigativa sociocrítica, con el cual pueden definirse acciones transformativas en la praxis de los sujetos e incluir a otras organizaciones en el desarrollo de estas perspectivas que favorecen y fortalecen el carácter colaborativo para la participación de las poblaciones vulnerables en el quehacer político y la construcción de ciudad.

\section{Bibliografía}

Borja, J. (2003). La ciudad conquistada. Madrid: Alianza Editorial.

Bronfenbrenner, U. (1987). La ecología del desarrollo humano. Barcelona: Paidós.

Congreso de Colombia. (27 de febrero de 2013). Ley Estatutaria 1618.

Díaz Velásquez, E. (2010). Ciudadanía, identidad y exclusión social de las personas con discapacidad, en: Politica y Sociedad. Volumen $47\left(\mathrm{~N}^{\circ} 1\right)$.

Egea García, C. y Sarabia Sánchez, A. (s.f.). Visión y modelos conceptuales de la discapacidad. Recuperado el 12 de octubre de 2015, de DISCATIF: http://www.um.es/discatif/METODOLOGIA/Egea-Sarabia modelos.pdf

Foucault, M. (2006). Seguridad, territorio, población: curso en el College de France (19771978). Buenos Aires: Fondo de Cultura Económica.

Geertz, C. (1973). Descripción densa: hacia una teoría interpretativa de la cultura. Barcelona: Gedisa.

Goffman, E. (2006). Estigma. La identidad deteriorada. Buenos Aires: Amorrortu. 
González Escobar, L. (2007). Del higienismo al Taylorismo: de los modelos a la realidad urbanística de Medellín, Colombia 1870-1932. Bitácora Urbano Territorial, 1, 11, pp. 149-159.

Harvey, D. (1979). Urbanismo y Desigualdad Social. Madrid: Siglo XXI.

ICONTEC. (25 de junio de 2008). Tienda ICONTEC. Recuperado el 11 de febrero de 2016, de http://tienda.icontec.org/brief/NTC5610.pdf

Iwarsson, S. y Stahl, A. (2003). Accessibility, usability and Universal Design -positioning and definition of concepts describing person-environment relationships. Disability and Rehabilitation, 25, 2, pp. 57-66.

Lefebvre, H. (1969). El derecho a la ciudad. Barcelona: Ediciones Península.

Lefebvre, H. (2013). La producción del espacio. Madrid: Capitán Swing.

Marshall, T. H. y Bottomore, T. (1998). Ciudadanía y Clase Social. Madrid: Alianza Editorial.

Montañés Serrano, M. (2009). Metodología y técnica participativa: Teoría y práctica de una estrategia de investigación participativa. Barcelona: UOC.

OMS. (2001). Clasificación Internacional de Funcionamiento, de la discapacidad y de la salud. Madrid: Ministerio de Trabajo y Asuntos Sociales. Instituto de Migraciones y Servicios Sociales (IMSERSO).

OMS. (2004). RBC: estrategia para la rehabilitación, la igualdad de oportunidades, la reducción de la pobreza y la integración social de las personas con discapacidad. Ginebra: Organización Mundial de la Salud.

ONU. (13 de febrero de 2007). Convención Internacional sobre los Derechos de las Personas con Discapacidad. Recuperado el 1 de agosto de 2014, de http://www.un.org/esa/socdev/enable/documents/tccconvs.pdf

Red CIMAS. (2015). Metodologías Participativas. Sociopraxis para la creatividad social. Madrid: Dextra.

Rodríguez, G.; Gil Flores, J. y García Jiménez, E. (1996). Metodología de la Investigación Cualitativa. Málaga: Aljibe.

Semillero de Investigación Observatorio de la Sociedad Civil. (2007). Incidencia de la participación de la sociedad civil en la construcción de la política social de discapacidad en Medellín. En Fernández Agudelo, M.; Otalvaro Orrego, J.; Giraldo, M.; Cano Chica, V.; Grisales Velásquez, M.; Ochoa López, D. y otros Incidencia de la participación de la sociedad civil en la construcción de la política social de discapacidad en Medellín. Medellín: Universidad de Antioquia. 
Tabla de Distribución por tipo de Discapacidad

\begin{tabular}{|l|l|}
\hline $\begin{array}{c}\text { Incluya su discapacidad en las } \\
\text { siguientes categorías: }\end{array}$ & Cantidad \\
\hline 1. Movilidad & 9792 \\
\hline 2. Sensorial Auditiva & 1356 \\
\hline 3. Sensorial Visual & 1666 \\
\hline 4. Sensorial Gusto-Olfato-Tacto & 35 \\
\hline 5. Sistémica & 1086 \\
\hline 6. Mental Cognitivo & 6948 \\
\hline 7. Mental Psicosocial & 2928 \\
\hline 8. Voz y Habla & 216 \\
\hline 9. Piel, Uñas y Cabello & 58 \\
\hline 10. Enanismo & 45 \\
\hline 11. No la sabe nombrar & 364 \\
\hline 12. Múltiple & 10091 \\
\hline En blanco & 24448 \\
\hline Total general & 59033 \\
\hline
\end{tabular}

Fuente: Registro de Localización y Caracterización de Personas con discapacidad, 17 de marzo de 2016

\section{Anexo}

\section{Imagen 1}

Validación en campo del Instrumento para la Verificación de la Accesibilidad (IVACCES)

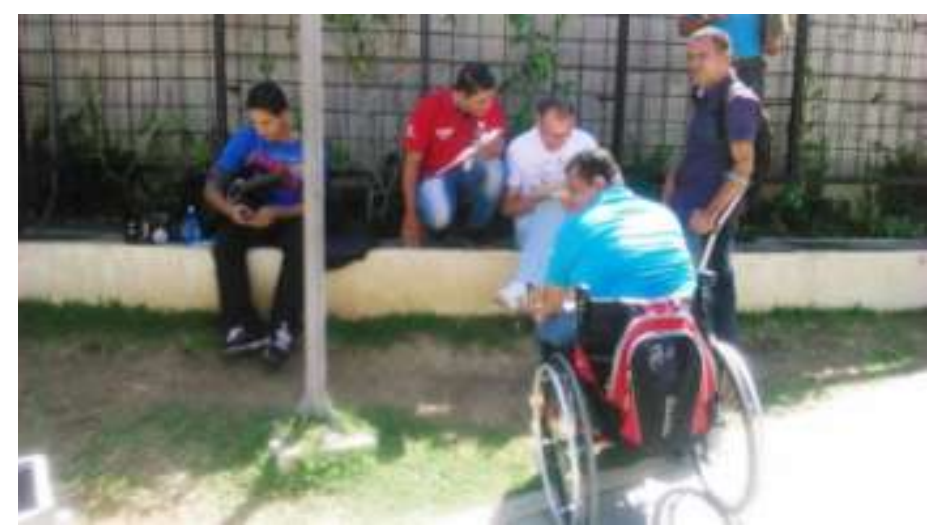




\section{Anexo}

\section{Imagen 2}

Diseño de Sociograma y Mapa de actores para la incidencia de la mesa de Accesibilidad

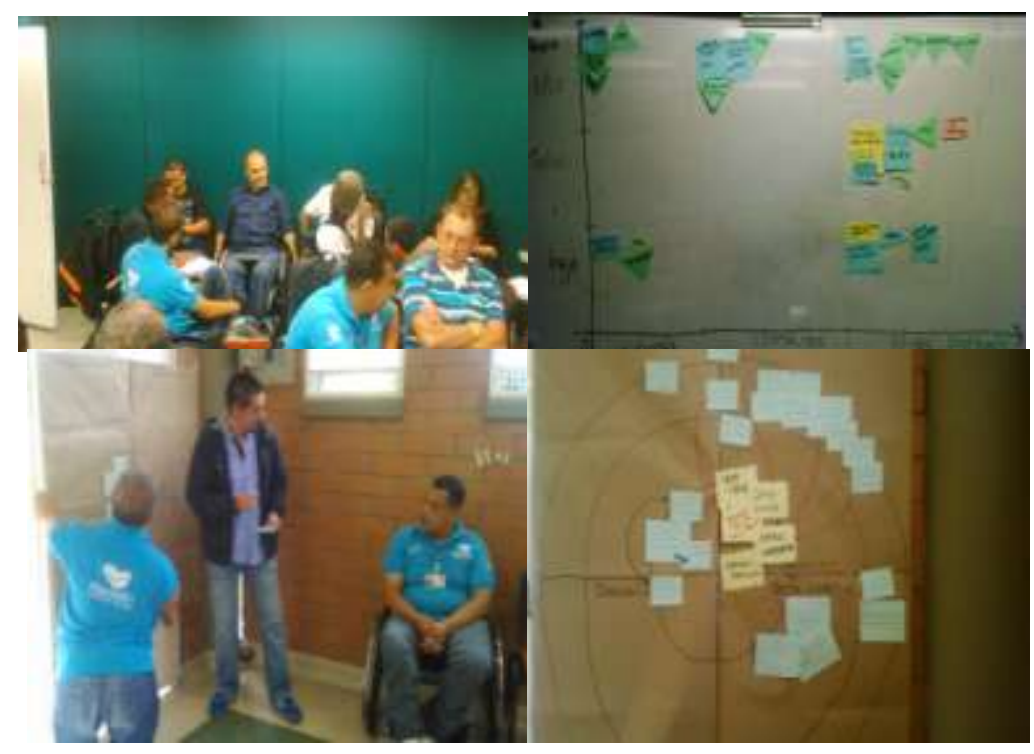

\section{Anexo}

\section{Imagen 3}

Recorridos y reconocimiento de espacios públicos recién inaugurados

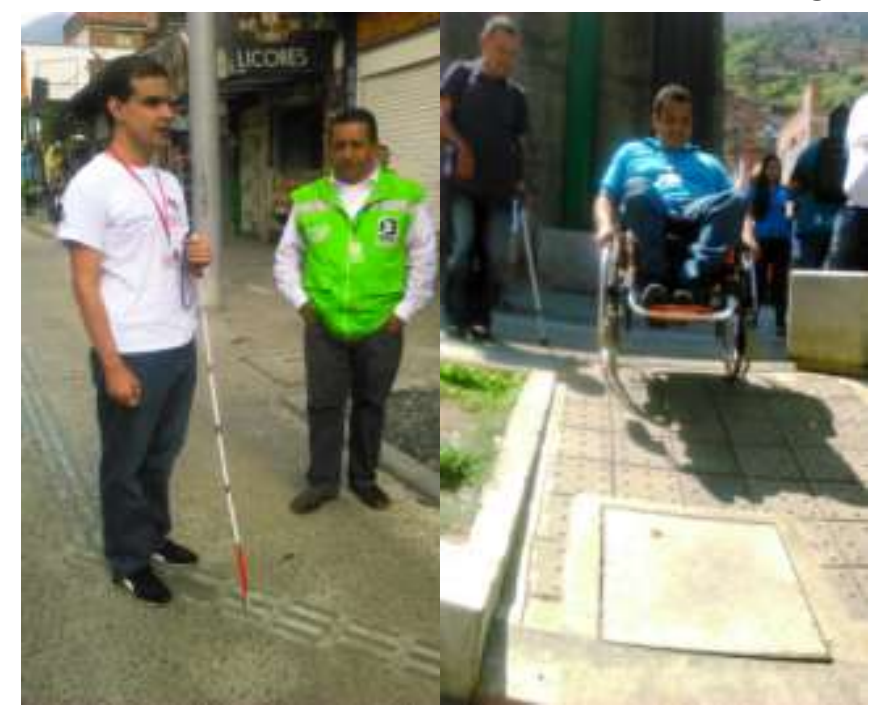

